\title{
Primary biliary cirrhosis
}

\author{
J Neuberger, M Lombard, R Galbraith
}

During the last 25 years there have been considerable advances in the understanding of primary biliary cirrhosis. It is now appreciated that the disease has a much wider clinical spectrum than previously thought, with many people being diagnosed in an asymptomatic state. The increasing success of liver transplantation has resulted in a greater need for accurate assessment of prognosis. Development of accurate prognostic models to assess survival has been aided by multicentre studies where large cohorts of patients have been followed for many years. Many immunological abnormalities associated with the disease have been identified: the diverse nature of the antimitochondrial antibodies - the serological hallmark of the disease - is recognised and the subtypes are being increasingly identified. The antigen, pyruvate dehydrogenase complex, has been defined and the antigenic epitopes are being defined. Yet, despite these major advances over the last quarter century, the pathogenesis of primary biliary cirrhosis remains elusive. Failure to define the pathogenesis of disease has resulted in a lack of a logical approach to therapy. Despite the increasing number of trials being carried out, no drug has yet shown to be totally effective in preventing progression of the disease.

\section{Clinical features}

Perhaps one of the greater strengths of the Liver Unit in the field of primary biliary cirrhosis has been the ability to collect and monitor a large series of patients with a comparatively rare disease. There has been a longstanding collaboration between the Liver Unit at King's College Hospital, the Division of Hepatology in the Rigshospitalet, Copenhagen and the Liver Unit in the Hospital Clínic i Provincial, Barcelona. The first landmark paper describing the clinical course of primary biliary cirrhosis appeared in $1980^{\prime}$ and was followed by a review of presenting symptoms five years later. ${ }^{2}$ Of the presenting symptoms, $47 \%$ presented with pruritus, $22 \%$ presented with non-specific symptoms, mainly tiredness, fatigue and right upper quadrant pain. Nineteen per cent presented with hepatic decompensation - jaundice, gastrointestinal bleeding or ascites. In $5 \%$ the presenting symptoms were suggestive of vascular disease or auto immune disease (such as arthritis, scleroderma, or Raynaud's phenomenon). It was of interest that even at that time $7 \%$ were identified who presented with no specific hepatological symptoms. Modes of presentation of this asymptomatic group included abnormalities on routine blood testing, or during investigation for other diseases such as diabetes mellitus. Follow up studies suggested that during first five years after presentation, jaundice increased from $59 \%$ to $82 \%$ and hepatomegaly from $54 \%$ to $86 \%$.
Gastrointestinal bleeding increased from $17 \%$ to $46 \%$ and ascites from $7 \%$ to $49 \%$. Cirrhosis increased more rapidly from $30 \%$ to $82 \%$ in four years.

Analysis of risk factors at that time suggested that age, histological stage, and most importantly serum bilirubin were of major prognostic value. Although serum immunoglobulins and titres of antimitochondrial antibody tended to rise with time, and ascites and gastrointestinal bleeding became more common, these were of less prognostic importance. During the study 36 patients died and perhaps not surprisingly, over $75 \%$ of these patients had hepatomegaly, jaundice and on histology, peripheral cholestasis and cirrhosis. One other feature of note was the poor correlation of symptoms and biochemical abnormalities with histological staging. Although, as might be anticipated those with more advanced histological stage did have more evidence of decompensation and a higher serum bilirubin, of those with histological stage one $(14 \%$ of the whole series) $39 \%$ had jaundice and $11 \%$ gastrointestinal bleeding; equally, $9 \%$ required treatment with diuretics.

Despite the marked female preponderance of the disease (about $90 \%$ ), the clinical course of disease in the two sexes appears similar. ${ }^{3}$ Although there was no difference in survival, it was observed that men present less commonly with pruritus and more commonly are detected on routine blood tests. While the latter may reflect merely the increased use of health screening in men, the former is of interest in particular when it was found that premenstrual women had more pruritus than postmenstrual women. The cause of pruritus in primary biliary cirrhosis is not understood; although recent work suggests that the endogenous opiate agonists ligands (such as encephalins) may be involved, ${ }^{4}$ the sex differences raise the possibility that sex steroids may modify response to pruritus.

With the increasing use of auto analysers, the greater availability of auto antibody testing, and the generally greater awareness by pathologists and clinicians of the possibility of primary biliary cirrhosis, appreciation of the clinical spectrum has widened dramatically. The term 'asymptomatic primary biliary cirrhosis' was introduced by Fox in 1973 and achieved much greater publicity after the review by James et al..$^{5} \mathrm{~A}$ large study formed the basis of a retrospective analysis of 95 patients with asymptomatic primary biliary cirrhosis in collaboration between the Liver Unit and Newcastle. ${ }^{6}$ The majority of these patients had early disease histologically assessed, but contrary to some reports, it was found these patients do progress clinically and histologically. Thirty five per cent of patients become symptomatic and the survival of these patients was no different from those who present with symptomatic disease and 15 of these died of liver related

Ashley Avenue,

Carolina, USA

R Galbraith

Correspondence to:

Dr J Neuberger. 
causes. Mortality was significantly greater than the normal age and sex matched population. Thus, although asymptomatic disease may represent a more benign end of the spectrum, there can be little doubt that there is progression in many patients. Overall, there does appear to be a wide variation in the rate of progression between patients.

Regarding side effects of the disease, bone disease can induce major morbidity and indeed mortality..$^{7}$ Osteoporosis may result from either cortical or trabecular bone loss. The latter is more important as vertebral, radial, and femoral neck fractures may result. Stellon in conjunction with Juliette Compston studied this problem in detail. Of 36 patients with cholestasis, $42 \%$ had symptoms suggestive of osteoporosis and $11 \%$ had one or more wedged crushed vertebrae. Compared with age and sex matched controls, $28 \%$ had excessive cortical and $36 \%$ excessive trabecular bone loss. Not surprisingly, bone loss was greatest in those who had received corticosteroids. In contrast, in this and most other series, osteomalacia rarely occurs, and vitamin D deficiency is associated mainly with prolonged cholestasis.

Further insight was gained by histological studies after double tetracycline labelling. The combination of increased osteoblastic function, decreased mineral apposition rate and prolonged mineralisation lag time suggest a defect in matrix synthesis. Additional studies suggested increase in resorption may also contribute to bone loss in primary biliary cirrhosis. The cause of this major complication remains uncertain. It may relate to the effects of cytokines which in vitro affect osteoblastic activity. Treatment remains uncertain: avoidance of corticosteroids, use of vitamin D supplements in association with calcium supplements and sodium fluoride have been advocated. There is no universal agreement for optimal treatment.

Of the other features associated with primary biliary cirrhosis, thyroid disorders and its association with thyroid autoantibodies and the Sicca syndrome, ${ }^{9}$ scleroderma, ${ }^{10}$ the incidence and pattern of rheumatic disorders ${ }^{11}$ and the association with increasing levels of circulating immune complexes ${ }^{12}$ and menstrual abnormalities ${ }^{13}$ have been reported.

Hepatocellular carcinoma was initially thought to be rare in this disease but a retrospective study of patients who developed primary biliary cirrhosis and hepatocellular carcinoma revealed that these patients are at risk of this complication. ${ }^{14}$ The development of hepatocellular carcinoma was often insidious and associated with the general progression of the disease. Serum alphafetoprotein was often raised and was sometimes the only antimortem evidence of malignant transformation. The pro-

TABLE I Problems with trials of therapy in primary biliary cirrhosis

Unknown aetiology

Long natural history

Uncertain progression

Withdrawal for transplantation Choice of end point portion of men to women developing hepatocellular carcinoma was similar to that in other forms of cirrhosis, emphasising work from the Unit and others, that duration of cirrhosis and male sex are the major risk factors for hepatoma development in United Kingdom.

\section{Histological features}

The morphological and histological features of primary biliary cirrhosis have been well described. The initial, often focal lesion of chronic non-suppurative destructive cholangitis primarily affects bile ducts of $50-100 \mu \mathrm{m}$ diameter. The cellular infiltrate is often mixed but $T$ lymphocytes predominate. In the normal liver, biliary epithelium may express HLA class I antigens but rarely class II antigens. Increased class II expression may suggest that biliary epithelial can present antigen but there is little evidence as yet that this can occur. Increased class II expression occurs in other diseases, such as sclerosing cholangitis and graft versus host disease, and thus may be a secondary phenomenon. The availability of liver histology from the follow up patients allowed analysis of the sequential and diagnostic features of primary biliary cirrhosis. In association with Hans Popper, 605 liver biopsy specimens from 209 patients with primary biliary cirrhosis were analysed. ${ }^{15}$ Four varieties of piecemeal necrosis (biliary, lymphocytic, ductular, and fibrotic) were identified. Lymphocyte piecemeal necrosis, usually identified with lobular hepatitis, was found in histological stage 2 and 3, whereas biliary piecemeal necrosis was present in stages 2,3 , and 4 . The fibrotic biliary piecemeal necrosis appeared only in the late cirrhotic stages. Although cholestatic features dominated, in $23 \%$ there were histological features compatible with overlap with chronic active hepatitis. These overlap features tended to be maintained throughout the course of the disease.

When the biopsies of patients who died were analysed, features suggesting rapidly progressive disease included the development of cirrhosis, increasing cholestasis, especially in association with prominent hyaline inclusions, abundance of coarse collagen fibres and 'halo formation' at the margin of fibrous septa. The controversial report of an association of granulomas with worse prognostic disease was not supported by this study. Again the problems of the conventional staging and lack of correlation with clinical features were described.

\section{Therapy}

The development of logical strategies for therapy is dependent on knowledge of the pathogenesis of the disease (Table I). The relatively slow progression of the disease makes drug trials difficult. Ultimately, the endpoint of any study must be either death or transplantation. Even alteration of known prognostic variables such as serum bilirubin does not necessarily mean that the prognosis has been altered. The natural history of the disease, the variability in presentation and the development of complications such as ascites or variceal haemorrhage means 
that large numbers of patients have to be studied and followed for many years. Indeed, based on an analysis of placebo patients in the various drug studies, it has been calculated that if a drug is associated with a $50 \%$ reduction in mortality, over 200 patients have to be followed for five years to show such an effect with statistical confidence. This has considerable logistic implications which will be fully appreciated by anyone who has been involved in drug studies!

The many immunological abnormalities associated with the disease have suggested that immune mechanisms are important in the pathogenesis and perpetuation of the disease and indeed primary biliary cirrhosis has been suggested to be an autoimmune disease. Early studies with corticosteroids showed little effect and their use has not been advocated because of the risk of exacerbating osteoporosis. More recent studies, however, have suggested that serum biochemistry may be improved by use of corticosteroids but the effect on bones remains uncertain. ${ }^{1617}$ A multicentre prospective, placebo controlled double blind study was set up to study the effect of azathioprine in 248 patients recruited from Europe and followed for up to 10 years. ${ }^{18}$ This study has proved a landmark for a number of reasons. Although the overall mortality in the two groups of patients was not significantly different, there was a slight but important imbalance between the two groups, in that those randomised to receive azathioprine had significantly more advanced disease. Correction for these imbalances suggested that azathioprine was associated with a small but significant improvement in survival (azathioprine reducing the mortality risk to 59\% of that observed in the placebo group - 95\% confidence intervals $40-90 \%)$. This would translate to an average improved survival of 20 months. Furthermore, azathioprine was associated with a reduction in the rate of symptomatic deterioration (Fig 1). An additional advantage from this study was the development of prognostic models for survival (see below).

At the time the study was concluding, preliminary reports were appearing in the literature suggesting that D-penicillamine was associated with reduced mortality, especially in those patients treated with stage 3 and 4 disease. This

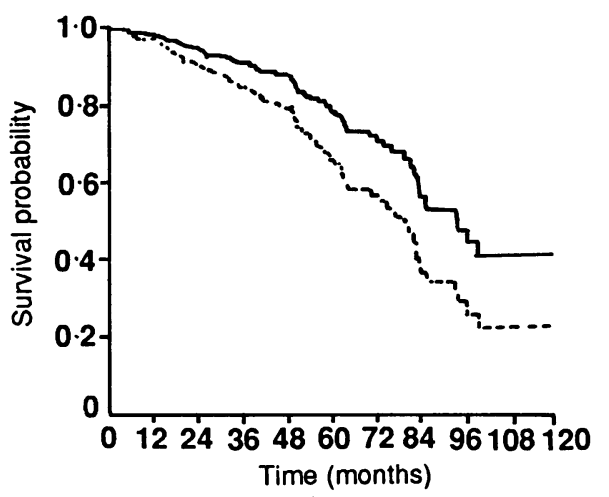

AZA: $1129276 \quad 64 \quad 55 \quad 44 \quad 30 \quad 19 \quad 17 \quad 13 \quad 9$ No of PLAC: $104847159 \quad 47 \quad 30 \quad 18 \quad 11 \quad 5 \quad 4 \quad 0$ patients

Figure 1: Survival probability in patients with primary biliary cirrhosis in controls and receiving azathioprine (from ref 19).
TABLE II Specific therapy for survival in primary biliary cirrhosis

\begin{tabular}{ll}
\hline Ineffective: & D-Penicillamine \\
Uncertain benefit: & Corticosteroids \\
& Ursodeoxycholic acid \\
Limited benefit: & Azathioprine \\
& Cyclosporin A \\
& Colchicine \\
& Methotrexate \\
Proven benefit: & Transplantation \\
\hline
\end{tabular}

prompted the start of a new study ${ }^{22}$ where D-penicillamine at a dose of $1200 \mathrm{mg} /$ day was assessed. One hundred and eighty nine patients entered the study and were followed for up to four years. Analysis showed no significant difference in survival between the two groups and there were no significant differences in disease state between the two groups. Indeed the only significant difference observed in the study was the withdrawal due to drug related side effects ( $36 \%$ against $89 \%$ ). The lack of response to D-penicillamine has also been supported by other studies. ${ }^{2324}$

In the last few years there have been reports of trials with a number of different agents (Table II). These include colchicine, methotrexate, chlorambucil, malotilate, ursodeoxycholic acid, and cyclosporin..$^{25-35}$ Of these, chlorambucil was suggested to be effective but too toxic. Methotrexate has been suggested to return liver tests to normal in some cases, but there are, as yet, no longterm controlled studies. Ursodeoxycholic acid (UDCA) has achieved much notoriety. There is an overall consensus that the drug often relieves pruritus and may improve liver function tests; one study ${ }^{30}$ suggested that the biochemical improvement was only transient. Results are awaited of longterm placebo controlled studies. In none of these studies has there been any clear cut effect on survival although much emphasis has been placed on differences in serum bilirubin and immunoglobulins between the two groups. Whether a reduction in serum bilirubin is associated with improved mortality has yet to be shown.

Cyclosporin corrects many of the immunological abnormalities in primary biliary cirrhosis, ${ }^{29}$ but a preliminary study using a dose of $10 \mathrm{mg} / \mathrm{kg} /$ day was discontinued because of nephrotoxicity. Smaller doses may be more effective. ${ }^{3233}$ The Liver Unit, continuing its well established collaboration with Barcelona and Copenhagen have just analysed a trial assessing cyclosporin $3 \mathrm{mg} / \mathrm{kg} / \mathrm{day} .{ }^{36}$ Three hundred and forty nine patients were followed for up to six years. Results were similar to the azathioprine study although survival was longer. Cox regression analysis showed cyclosporin prolongs survival to death and transplantation by $60 \%$. Whether further analysis will show there is a benefit in one particular subgroup, such as those with early stage disease, has yet to be shown. Cyclosporin is associated with a significant incidence of side effects, notably renal impairment (9\%) and hypertension (11\%). Whether this, together with the cost will preclude its greater use in primary biliary cirrhosis remains to be ascertained.

Overall, specific treatment for primary biliary 
cirrhosis remains far from satisfactory. The number of different agents tried reflects the growing desperation to find a cure for this enigmatic disease.

\section{Prognostic models}

The increasing use of liver transplantation and its acceptance as a therapeutic modality for endstage disease has led to new emphasis being placed on prognostic models. Because indications for liver transplantation include an estimated survival in the absence of transplantation of one year or less, the ability to define and quantify anticipated survival has become increasingly important. The first attempt at quantification of survival was developed from data gathered in the 1985 azathioprine trial. ${ }^{19}$ The model developed included six variables: the major component was serum bilirubin but other factors included age, serum albumin, histological presence of cirrhosis and central cholestasis and whether the patient was treated with azathioprine. By entering the six variables into a formula (Table III), a prognostic index was derived and from this the one year survival probability or the $50 \%$ survival probability can be estimated (Fig 2). The model was further validated by comparing prognostic data with actual data in a separate group of placebo patients, followed as part of the penicillamine study. Since then, several other groups have also developed prognostic models using different criteria $^{2021}$ and it is of interest that the models correlate well. It is of interest that in almost all models, bilirubin, age, and albumin are major components.

It must be remembered that all these models concentrate only on estimates of prognosis derived from data at referral or diagnosis. It is inappropriate to use these models in a time dependent analysis. Indeed, preliminary data analysed by Christensen suggest that different factors are significant prognostic factors in time dependent models.

The ability to give an objective and quantitative estimate of survival allows an objective method of audit of the results of transplantation. Preliminary analysis ${ }^{35}$ showed that there was a significant improvement in survival compared with anticipated survival in the absence of such procedure. This result has been subsequently confirmed by other centres. ${ }^{37} 38$ Prognostic factors for survival after transplantation are not the same as those indicating prognosis in the absence of transplantation (Table IV). ${ }^{39}$

\section{Does placebo biliary cirrhosis recur after transplantation?}

It has been appreciated for many years that the antimitochondrial antibodies persist after orthotopic liver transplantation. ${ }^{+0}$ It is far more controversial, however, as to whether the disease recurs in the transplanted liver. An initial report from the Liver Unit ${ }^{41}$ shows that in a small number of patients followed for up to five years, there were clinical, serological and histological features of disease recurrence. These findings were reinforced by a subsequent publication ${ }^{42}$

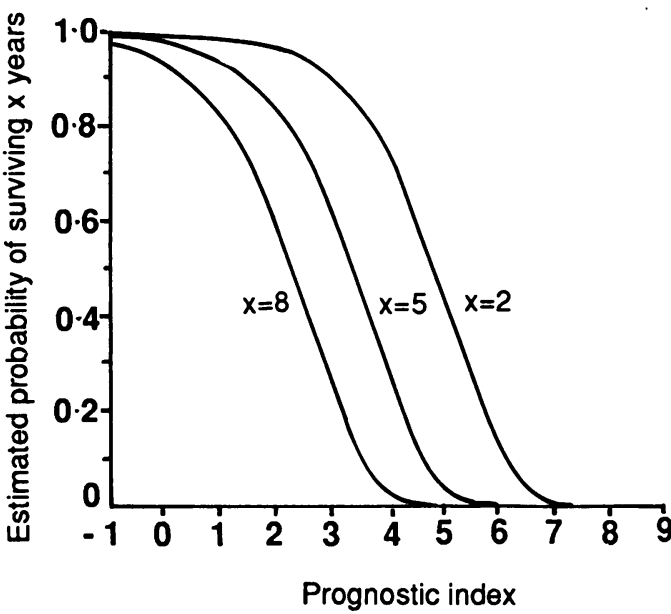

Figure 2: Estimated survival probability for surviving two, five or years (from ref 19).

but they have not been confirmed by other centres: ${ }^{43}$ while there is uniform agreement that mitochondrial antibodies recur, the histological findings have suggested this is due to mild chronic rejection. This controversy has still yet to be resolved but with the increasing number of patients undergoing transplantation and the increasing survival and long follow up, it is anticipated that in the next few years this question will be resolved.

\section{Immunological abnormalities in primary biliary cirrhosis}

In contrast with autoimmune chronic active hepatitis, there is no clear class I or class II HLA association with primary biliary cirrhosis although occasional reports have suggested increased incidence of DRW3 and DRW8. The Liver Unit study did suggest an increased frequency of the class III phenotype C4B2 but not of other class I or II phenotypes. ${ }^{44}$ Because these phenotypes are all found in approximately half the patients with disease, it is unlikely that any of these phenotypes represent a major genetic influence. It is, however, of interest that C4B2 has been associated with other chronic diseases with a presumed viral aetiology and DR3 with autoimmune diseases. A genetic association may

TABLE III Prognosis for survival of primary biliary cirrhosis

$2.52 \times \log$ serum bilirubin $(\mu \mathrm{mol} / \mathrm{l})$

$\begin{aligned} & 2 \cdot 52 \times \log \text { serum bilirubin }(\mu \mathrm{m} \\ + & 0.0069 \times \exp ((\text { age }-20) / 10)\end{aligned}$

$+\quad 0.0069 \times \exp (($ age -20$) / 10)$
$-\quad 0.05 \times$ serum albumin $(\mathrm{g} / \mathrm{l})$

$-0.05 \times$ serum albumin $(g / 1)$
$+\quad 0.88$ (if cirrhosis present)

$+\quad 0.88$ (if cirrhosis present)
$+\quad 0.52$ (if not treated with azathioprine)

(a PI of 6 implies a median prognosis of 10 months and of 5 at 22 months).

TABLE IV Prognosis for surviving six months after transplantation for primary biliary cirrhosis

$1.89 \times \log$ serum bilirubin $(\mathrm{mmol} / \mathrm{l})$

$+1.38 \log$ serum bilirubin/ $\mu \mathrm{mol} / 1$

- $1 \cdot 14$ (if transplanted before 1985 )

- 0.92 (if patient has diuretic responsive ascites)

(a PI of 4.0 implies an $80 \%$ and of 5 a $42 \%$ probability of surviving six months). 
also explain the rare occurrence of disease in families.

Of the serological abnormalities, the association with raised serum IgM, monomeric IgM, total and secretory $\operatorname{IgA}$, and polyclonal $\operatorname{IgG}$ is well recognised. None of these findings is specific to primary biliary cirrhosis. Almost diagnostic of the disease is the presence of the antimitochondrial antibody. The antigen is neither liver nor bile duct specific. There is little evidence it is present on the plasma membrane. Inoculation of antigen in a variety of animals induces anti-E2 but without evidence of liver disease. Other autoantibodies have been described in primary biliary cirrhosis: these include nuclear and nucleolar antibodies and anticentromere antibodies which are strongly associated both in primary biliary cirrhosis and in other conditions with sclerodactyly. Immune complexes are increased and there is hypercatabolism of complement. ${ }^{45-47}$

Nine different antimitochondrial antibodies have been identified and some of these may be detected in a number of diseases, including drug induced disorders and some infective disorders. Primary biliary cirrhosis is associated especially with $M 2, M 4, M 8$, and M9; it has been suggested that the different subtypes are associated with varying prognosis. M2 recognises an antigen on the inner mitochondrial membrane, the remainder react with antigens on the outer membrane. There is confusing information whether the antigens are present on the surface membrane of cells. ${ }^{48}$

Four of the major M2 autoantibodies are the E2 components of three 2-oxo-acid dehydrogenase complex and protein $x$ of the pyruvate dehydrogenase complex, an enzyme system present in mitochondrian but encoded in the nucleus. The enzymes are almost universal and are well conserved. Despite the major advances in defining the antigens of the antimitochondrial antibodies system, their significance remains to be established. The universal distribution of pyruvate dehydrogenase complex in all tissues contrasts with the focal nature of disease and the liver. Many bacterial organisms contain pyruvate dehydrogenase complex, therefore, it is possible that a cross reaction may trigger the immune response.

Because of the association with other autoimmune diseases and the immune abnormalities, much work has concentrated on examining immunoregulatory cell function. A number of centres have shown that peripheral lymphocytes have impaired suppressor cell activity as tested by concanavalin A induced suppression of mitogen stimulated immunoglobulin synthesis. ${ }^{49}$ This suppressor cell activity is affected in vitro by corticosteroids, colchicine, and cyclosporin. Monocyte function is also abnormal; possibly a consequence of circulating factors such as immune complexes. Many of these immunological abnormalities, including the presence of antimitochondrial antibodies, are found in a significant proportion of relatives of patients with primary biliary cirrhosis even though they may have no clinical evidence of disease..$^{50}$

In contrast with these relatively non-specific peripheral immunologic abnormalities, other work led by the McFarlanes in the Liver Unit has concentrated on organ specific biliary tract antigens. ${ }^{5152}$ Three antigens, which are speciesspecific high molecular weight carbohydrate or lipid containing protein components, have been identified in normal human hepatic and gall bladder bile. Of these three antigens: one (antigen 1) has not been localised to a particular structure of the liver and is not specific to patients with primary biliary cirrhosis. This may represent an acute phase protein. The second antigen, the canalicular antigen, appears to be derived from the bile biliary canalicular portion of the hepatocellular membrane. The ductular antigen is derived from the epithelial cell lining the intrahepatic bile ducts. Only the latter antigen is specific to the biliary tree. Most patients with primary biliary cirrhosis show cellular immune responses to these antigens and it is of interest that the immune complexes in serum from patients with the disease contain one or more of these antigens. Whether these antigens and their responses in patients with primary biliary cirrhosis represent a primary or secondary immunological event remains far from clear. It is unfortunate that the technical problems in isolating these antigens has precluded further investigations into what remains a novel approach to investigate the disease. Recent studies have suggested that lymphocytes from patients with primary biliary cirrhosis, injected with immune deficient mice can induce a primary biliary cirrhosis-like lesion in the liver. ${ }^{53}$ If repeated, these results confirm the importance of the search for primary biliary cirrhosis specific antigens on middle sized bile ducts.

\section{The future}

The past 25 years has seen major changes in our understanding of primary biliary cirrhosis. Despite increasing laboratory work revealing yet more abnormalities especially of the immunological system, understanding of the pathogenesis of the disease is still limited.

One of the major strengths of the Liver Unit has been its ability to mount and coordinate major international studies which have given clear cut answers to the efficacy of different drugs. As yet, no drug has been identified which has a major effect on the progression of the disease. Use of data derived from these trials has allowed generation of prognostic models to optimise the timing of orthotopic liver transplantation. Continuing collaborative studies must be the way forward to confirm or refute therapeutic efficacy of drugs. Primary biliary cirrhosis remains the most common single indication for liver transplantation in Europe and it is to be hoped that therapeutic regimes can be designed on the basis of the understanding derived from work during the past quarter of century to make primary biliary cirrhosis a disease no longer requiring such radical therapy.

Figures 1 and 2 are reproduced with kind permission of Gastroenterology.

1 Christensen E, Crowe J, Doniach D, et al. Clinical pattern and course of disease in primary biliary cirrhosis. Gastroenterology 1980; 78: 236-47. 
2 Crowe J, Christensen E, Doniach D, Popper H, Tygstrup N, Williams R. Presentation of PBC. Am f Gastroenterol 1985; 80: 466-8.

3 Lucey MR, Neuberger J, Williams R. Primary biliary cirrhosis in men. Gut 1986; 27: 1373-6.

4 Jones EA, Bergasa NV. The pruritus of cholestasis: from bile acids to opiate agonists. Hepatology 1990; 11: 884-7.

5 James OFW, Macklin AF, Watson AJ. Primary biliary cirrhosis - a revised clinical spectrum. Lancet 1981; i: 1278-81.

6 Mitchison HC, Lucey M, Kelly P, Neuberger J, Williams R, James OFW. Symptom development and prognosis in primary biliary cirrhosis. Gastroenterology 1990; 99: 778-84.

7 Stellon AJ, Davies A, Compston J, Williams R. Osteoporosis in chronic cholestatic liver disease. $Q \mathcal{F}$ Med 1985; 223:

turnover state in primary biliary cirrhosis. Hepatology 1987;
, 7: $137-42$.

9 Crowe JP, Christensen E, Butler J, et al. Primary biliary cirrhosis: the prevalence of hypothyroidism and its relationship to thyroid auto-antibodies and Sicca syndrome. Gastroenterology 1980; 79: 1437-41.

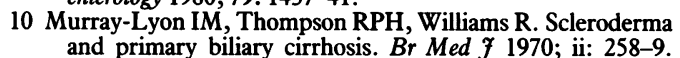

11 Clark AK, Galbraith RM, Hamilton EDB, Williams R. Rheumatic disorders in primary biliary cirrhosis. Ann Rheum Dis 1978; 37: 42-7.

12 Crowe JP, Malloy MG, Wells I, et al. Increased C1Q binding and arthritis in primary biliary cirrhosis. Gut 1980; 21: 418-22.

13 Stellon AJ, Williams R. Increased incidence of menstrual abnormalities and hysterectomy proceeding primary biliary cirrhosis. BMF 1986; $293: 297$.

14 Melia WM, Johnson PJ, Neuberger J, Zaman S, Portmann B, Williams R. Hepatocellular carcinoma in primary biliary cirrhosis: detection by alphafetoprotein estimation. Gastroenterology 1984; 87: 660-3.

15 Portmann B, Popper H, Neuberger J, Williams R. Sequential and diagnostic features in primary biliary cirrhosis based on serial histological study in 209 patients. Gastroenterology 1985; 88: 1777-90.

16 Mitchison HC, Bassendine MF, Malcolm AJ, et al. A pilot, double blind controlled one year trial of Prednisolone treatment in primary biliary cirrhosis. Hepatology 1989; 10: treatmen.

17 Coombes B. Prednisolone for primary biliary cirrhosis - good news, bad news. Hepatology 1989; 10: 511-3.

18 Crowe J, Christensen E, Smith $M$, et al. Azathioprine in primary biliary cirrhosis. A preliminary report of an international trial. Gastroenterology 1980; 78: 1-7.

19 Christensen E, Neuberger JM, Crowe J, Doniach D, Popper $H$, Williams R. Beneficial effect of Azathioprine and prediction of prognosis in primary biliary cirrhosis. Gastroprediction of prognosis in prim
enterology 1985; 89: $1084-91$.

20 Neuberger J, Christensen E, Portmann B, et al. Double-blind controlled trial of d-penicillamine in patients with primary biliary cirrhosis. Gut 1985; 26: 114-9.

21 Roll J, Boyer JL, Berry D, Klatskin G. The prognostic importance of clinical and histological features in asymptomatic and symptomatic primary biliary cirrhosis. $N E n g l f$ Med 1983; 308: 1-7.

22 Dickson ER, Grambsch PM, Fleming TR, Fisher W, Longworthy N. Prognosis in primary biliary cirrhosis. Longworthy N. Prognos
Hepatology 1989; 10: 1-7.

23 James OFW. D-penicillamine for primary biliary cirrhosis. Gut 1985; 26: 109-13.

24 Bodenheimer HC, Schnaffner F, Sternleib I, et al. A prospective clinical trial of d-penicillamine in the treatment of primary biliary cirrhosis. Hepatology 1985; 5: 1139-94.

25 Kaplan MM, Alling DW, Wolfe HJ, et al. A prospective trial of colchicine for primary biliary cirrhosis. N Engl f Med 1989; 315: 1448

26 Warnes TW, Smith AM, Lee F. A controlled trial of colchicine for primary biliary cirrhosis. 7 Hepatol 1987; 5: 1-7.

27 Bodenheimer H, Schnaffner F, Pezzulo J. Evaluation of colchicine therapy in primary biliary cirrhosis. Gastroenterology 1988; 95: 24-129.

28 James OFW. Ursodeoxycholic acid treatment for chronic liver disease. 7 Hepatol 1990; ii: 5-8.
29 Poupon P, Poupon RE, Calmus Y, et al. Is ursodeoxycholic acid an effective treatment for primary biliary cirrhosis? Lancet 1987 ; i: $834-6$.

30 Hadzyannis SJ, Hadzyannis EJ, Makris A. A randomized controlled trial of ursodeoxycholic acid (UDCA) in primary biliary cirrhosis [Abstract]. Hepatology 1989; 10: 580.

31 Routhier G, Epstein O, Janossy G, et al. Effects of cyclosporin A on suppressor and induced T lymphocytes in PBC. Lancet 1980; ii: 1223-6.

32 Minuk G, Bohme C, Burgess E, et al. Pilot study of cyclosporin $A$ in patients with symptomatic primary biliary cirrhosis. Gastroenterology 1988; 95: 1356-63.

33 Weisner RH, Ludwig J, Klindon KD, et al. A controlled clinical trial of cyclosporin in the treatment of primary biliary cirrhosis. NEnglF Med 1990; 322: 1419-29.

34 Hoofnagle J, David GL, Schnafer D, et al. Randomized trial of chlorambucil for primary biliary cirrhosis. Gastroenterology 1986; 91: 1327-4.

35 Kaplan $M$. Methotrexate treatment of chronic cholestatic disease. Qf Med 1989; 268: 757-61.

36 Lombard $M$, Portmann R, Neuberger J, et al. Cyclosporin A treatment in primary biliary cirrhosis. [Abstract]. Hepatology 1990; 12: 872 .

37 Neuberger J, Altman DG, Christensen E, Tygstrup N, Williams $\mathbf{R}$. Use of a prognostic index in liver transplantation for PBC. Transplantation 1986; 41: 713-6.

38 Markus B, Dickson ER, Grambsch P, et al. Efficacy of liver transplantation in patients with primary biliary cirrhosis. transplantation in patients with $p$
$N E n g l$ f Med 1989; 320: $1709-13$.

39 Neuberger J, Altman DG, Polson R, et al. Prognosis after liver transplantation for primary biliary cirrhosis. Transplantation 1989; 48: 444-7

40 Smith MGM, Williams R, Doniach D, Calne RY. Effect of orthotopic liver transplantation on serum-auto-antibodies. Lancet 1971; ii: 1006.

41 Neuberger JM, Portmann B, Macdougall BRD, et al. Recurrence of primary biliary cirrhosis after liver transplantation. NEnglf Med 1982; 306: 1-4.

42 Polson RJ, Portmann B, Neuberger JM, Calne RY, Williams R. Evidence for disease recurrence after liver transplantation for PBC. Gastroenterology 1989; 97 : 715-25.

43 Esquivel CU, Van Thiel DH, Bernados A, et al. Liver transplantation for primary biliary cirrhosis. Gastroenterology 1982; 94: 1207-16.

44 Briggs D, Donaldson P, Hayes P, et al. A major histocompatability of complex class III allotype (CB2) associated with PBC. Tissue Antigens 1987; 29: 141-5.

45 James SP, Elson CO, Jones EA, Strober W. Abnormal regulation of immunoglobulin synthesis in primary biliary cirrhosis. Gastroenterology 1980; 79: 242-54.

46 Bernstein R, Neuberger J, Bunn C, Callensen M, Hughes G, Williams R. Diversity of auto-antibodies in primary biliary cirrhosis and chronic active hepatitis. Clin Exp Immunol 1984; 55: 553-60.

47 Wands J, Dienstag J, Bhan AK, Feller ER, Isselbacher KS. Circulating immune complexes and complement activation in primary biliary cirrhosis. $N$ Engl $\mathcal{F}$ Med 1978; 298: in primary

48 Al-Aghbar MNA, Alexander G, Nouri-Aria K, Neuberger J, Eddleston A, Williams $R$. In vitro effect of cyclosporin A on immunoglobulin products and concanvalin induced suppression in primary biliary cirrhosis. Gut 1986; 27: 317-23.

49 Van De Water J, Surch C, Leung PSC, et al. Molecular definitions, auto epitopes and enzymatic activities of the mitochondrial auto-antigens of primary biliary cirrhosis. Semin Liver Dis 1989; 9: 132-7.

50 Galbraith RA, Smith M, Mackenzie RM, et al. High prevalence of sero-immunologic abnormalities in relatives of patients with chronic active hepatitis or primary biliary cirrhosis. N Engl f Med 1974; 290: 63-9.

51 McFarlane G, Woicicka BM, Tsantoulas DL, et al Leucocyte migration inhibition in response to biliary antigens in primary biliary cirrhosis, chronic active hepatitis and other primary biliary cirrhosis, chronic active hepatitis

52 Amoroso P, Vergani D, Wojcicka B, Eddleston A, Tee D, Williams R. Identification of biliary antigens in circulating complexes in PBC. Clin Exp Immunol 1980; 42: 95-101.

53 Krams SM, Dorschkino K, Gershwin M. Generation of biliary lesions after transfer of human lymphocytes to severe combined immunodeficient (SCID) mice. $\mathcal{F}$ Exp Med 1989; 170: $1919-30$ 